\title{
COMMENTARIES
}

\section{Pandemic Dementia Scarce Resource Allocation}

Eric E. Smith, $\mathrm{MD}^{1}$; Philippe Couillard, $\mathrm{MD}^{2}$, John D. Fisk, $\mathrm{PhD}^{3}$, Zahinoor Ismail, $\mathrm{MD}^{4}$, Manuel Montero-Odasso, $\mathrm{MD}^{5}$, Julie M. Robillard, $\mathrm{PhD}^{6}$, Isabelle Vedel, $\mathrm{MD}^{7}$, Saskia Sivananthan, $\mathrm{PhD}^{8}$, Serge Gauthier, $\mathrm{MD}^{9}$

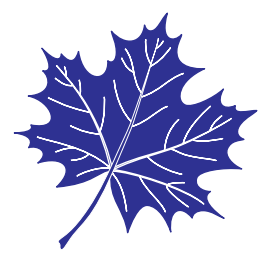

${ }^{1}$ Department of Clinical Neurosciences, Hotchkiss Brain Institute, University of Calgary, Calgary, AB;

${ }^{2}$ Department of Clinical Neurosciences and Department of Critical Care Medicine, Hotchkiss Brain Institute, University of Calgary, Calgary, AB; ${ }^{3}$ Nova Scotia Health Authority, Halifax, and Department of Psychiatry, Dalhousie University, Halifax, NS; ${ }^{4}$ Department of Psychiatry, Hotchkiss Brain Institute, University of Calgary, Calgary, AB; ${ }^{5}$ Departments of Medicine (Geriatrics), and Epidemiology and Biostatistics, The University of Western Ontario, London; Lawson Health Research Institute, Gait and Brain Lab, University of Western Ontario, London, ON; ${ }^{6}$ Department of Medicine, University of British Columbia, Vancouver; BC Children's \& Women's Hospital, Vancouver, BC; ${ }^{7}$ Department of Family Medicine, Mc Gill University, Montreal, QC; ${ }^{8}$ Alzheimer Society of Canada, Toronto, ON; ${ }^{9}$ Departments of Neurology, Neurosurgery and Psychiatry, McGill University, Montreal, QC, Canada https://doi.org/10.5770/cgj.23.457

\begin{abstract}
Hospitals and intensive care units are straining to provide care for a large surge of patients with coronavirus disease 19 (Covid-19). Contingency plans are being made for the possibility that resources for lifesaving care, including mechanical ventilators, will be in short supply. Covid-19 is more severe and more likely to be fatal in older persons. Dementia is one of the commonest severe comorbidities of aging. Persons with dementia are vulnerable and often need the support of others to make their voices heard. This commentary, created by a task force commissioned by the Alzheimer Society of Canada, provides guidance for triaging persons with dementia to scarce medical resources during the Covid-19 pandemic.
\end{abstract}

Key words: dementia, COVID-19, ethics, critical care, mechanical ventilation

The global pandemic of Covid-19 caused by Severe Acute Respiratory Syndrome Coronavirus-2 (SARS-CoV-2) is putting unprecedented strain on health-care systems. Even in high-income countries there are regional shortages of critical resources, including mechanical ventilators. ${ }^{(1)}$ The majority of the critically ill are older. In the United States, more than $80 \%$ of the deaths have occurred in persons $\geq 65$ years of age. (2) Because the prevalence of dementia rises with age, persons with dementia are expected to bear a disproportionate burden of severe illness compared to younger persons. Here, we provide principles for considering dementia as a criterion for access to life-saving care, including mechanical ventilation.
In the future, these principles may also guide access to scarce resources such as pharmaceutical treatments for COVID-19 and vaccines. The principles were generated by a task force commissioned by the Alzheimer Society of Canada that included experts in neurology, geriatrics, psychiatry, intensive care, ethics, and knowledge translation.

Dementia is a heterogeneous clinical syndrome with multiple causes and a variable trajectory. ${ }^{(3)}$ It is defined as an acquired decline in social and occupational functioning due to impairments in cognition. ${ }^{(4)}$ The diseases that most commonly cause dementia are Alzheimer's disease, vascular disease, and Lewy body disease, often in combination. Alzheimer's disease, the most common cause, has no disease-modifying treatments, and is progressive but can have a long disease duration (average 7-10 years ${ }^{(5)}$ ). Some causes of dementia, such as vascular disease, may be treatable and, thus, not all patients with dementia experience progression. Discriminating different causes of dementia is challenging using current diagnostic testing; therefore, caution should be exercised when making individual prognoses.

Dementia predominantly affects older persons. Thus, persons with dementia are at higher risk from Covid-19. The prevalence of dementia doubles every five years for Canadians 65 and older, rising from $1 \%$ of Canadians age 65-69 to $25 \%$ of Canadians age 85 or older. ${ }^{(6)}$ However, some younger persons are affected too. Of all Canadians with dementia, approximately $3 \%$ are under age $65 .^{(6)}$

The term "dementia" encompasses a wide range of severity. In its earliest stages, there are difficulties with more complex instrumental daily activities of living such as managing finances, shopping, complex household tasks (e.g., cooking), 
and driving. In the moderate stages, there are difficulties with basic daily activities like dressing and bathing. In the more severe stages, the person with dementia is dependent on others for daily activities. In Canada, most persons with dementia (61\%) live in the community. ${ }^{(6)}$ Social distancing and re-organizing of outpatient and facility-based care related to the Covid-19 pandemic are already having adverse effects on dementia care in the community. ${ }^{(7)}$

Currently, many regions are urgently planning triage systems for allocating care if the number of patients with Covid-19 exceeds the available resources. Ethical frameworks for resource allocation acknowledge that maximizing benefits is an important principle, and this favours allocation to persons with better likelihood of recovery, as well as younger persons with more years of life remaining. ${ }^{(8,9)}$ This principle of maximizing benefits is supported by community consultation. ${ }^{(10)}$ Previous planning for theoretical pandemics can be leveraged. ${ }^{(10)}$ However, because of regional variation in Covid-19 incidence, medical resources, and cultural attitudes, we expect that urgent planning is needed to operationalize prior theoretical exercises into actual principles for decision-making, relevant for local contexts.

Dementia affects older persons and increases the future risk of dying, and it is important to integrate these facts into resource allocation planning. However, given the variation in causes and trajectories of the conditions that cause dementia, the diagnosis per se does not reflect the wide range of function and physiological reserve experienced by persons with dementia.

To assist with resource planning, we suggest that the following principles should be considered when planning access to scarce resources to ensure that respect for the dignity of persons with dementia is preserved.

\section{Decisions on access to lifesaving resources for persons with dementia should be individualized.}

Dementia is a heterogenous syndrome with a wide range of severity and variation in individual trajectories. Persons with dementia live many years with good quality, given the average of 7-10 years' disease duration. Thus, decisions to provide, or withhold, lifesaving care for persons with dementia should be based on individual characteristics. Blanket restrictions on lifesaving care for persons with dementia are not appropriate. Persons with dementia should be treated in a similar fashion, using the same frameworks, as persons with other serious conditions such as heart failure and active cancer.

\section{Persons with dementia should be provided the opportun- ity to indicate their wishes regarding goals of care.}

All persons with dementia should have the opportunity to express their wishes, including whether they would wish to be resuscitated in the event of severe illness. This process is critical to support the ethical principle of autonomy, and ideally should be carried out in advance of serious illness. For persons who have lost the capacity to articulate their wishes, next-of-kin or legally designated representatives may be required to weigh in. However, given the evidence about discrepancies in quality of life evaluations between persons with physical or cognitive disabilities and their care partners, every effort should be made to engage persons directly about their wishes when possible.

\section{Decisions on resource allocation should respect the personhood of people with dementia.}

In an emerging pandemic where health-care needs exceed available resources, protocols to triage access to resources are needed. In response to this challenge and to address the unfortunate history of stigma against persons with dementia within the healthcare system, it is strongly encouraged to meaningfully engage with persons with lived experience and their care partners in developing these protocols for access to resources. The rapidly evolving circumstances may only allow limited engagement at first, but it should be expanded over time. Resource allocation protocols should treat persons with dementia in a similar fashion to persons with other health conditions, without discrimination. To reduce stigma and promote equity, it is preferable to define guiding principles in advance. The use of a triage committee could relieve individual clinicians of the burden of making life or death decisions and promote fair, equitable access.

\section{Decisions on access to lifesaving resources should be based on expected survival, not presumptions about in- dividual quality of life.}

Many persons with dementia report good quality of life that is sometimes underestimated by those around them, even their own care partners. ${ }^{(11)}$ Clinicians and health-care administrators should not assume that dementia is synonymous with intolerable suffering. Emanuel and colleagues ${ }^{(9)}$ offer the perspective that maximizing survival should be the primary objective in a pandemic situation, instead of attempting to predict future quality of life with limited time and information.

\section{Prognostications should be based on an objective, vali- dated assessment of mortality risk, frailty, or functional disease stage.}

When guidance principles for access to lifesaving resources are based on expected survival, they should be informed by best available evidence. Assessment should be based on a validated scale that is quick and reliable, and that can be implemented and understood by clinicians who are not dementia specialists. This reduces the heterogeneity and risk for bias that could creep into individual clinician judgements. Advancing dementia causes frailty, which can be measured using a simple global index such as the Clinical Frailty Scale $(\mathrm{CFS})^{(12)}$ which has been made available freely online. ${ }^{(13)} \mathrm{CFS}$ scores correlate well with future mortality. ${ }^{(12)}$ Although not commonly used in clinical practice and not intended for the purpose of determining access to care, there are mortality risk models that have been developed for persons with dementia. (14) Another reasonable alternative is to base decisions on dementia functional stage. ${ }^{(15)}$ 


\section{SMITH: PANDEMIC SCARCE RESOURCE ALLOCATION}

6. The presence of mild cognitive impairment should not be used as a criterion for assessing suitability for lifesaving care. Mild cognitive impairment (MCI), also called minor neurocognitive disorder, is a risk factor for developing of dementia, although many people with MCI remain stable or improve over time. ${ }^{(16)}$ Persons with MCI do not have disabilities and are able to live independently despite objective evidence of lower performance on cognitive testing.

7. Persons with dementia who are denied potentially lifesaving care are entitled to an explanation and best alternative care, including palliative care, if appropriate.

If the number of patients with severe Covid-19 illness exceeds the capacity of mechanical ventilators in Canada, it is likely that ventilatory support may be denied to some persons with dementia. To respect their personhood and preserve trust in the health system, patients and their care partners deserve a frank explanation for the reason that care is being limited, even though this conversation may be emotionally difficult for the care team. ${ }^{(9)}$ When care is limited, patients deserve the best alternative care, including access to good quality palliative care, as appropriate. ${ }^{(17)}$

These seven principles are suitable for patient care around the world and we hope that this opinion piece, commissioned by the Alzheimer Society of Canada and endorsed by a nationally representative multidisciplinary task force, will prove useful for patient care everywhere.

\section{ACKNOWLEDGEMENTS}

The work was funded by the Alzheimer Society of Canada.

\section{CONFLICT OF INTEREST DISCLOSURES}

The authors declare that no conflicts of interest exist.

\section{REFERENCES}

1. Ranney ML, Griffeth V, Jha AK. Critical supply shortages - the need for ventilators and personal protective equipment during the Covid-19 pandemic. N Engl J Med. 2020;382(18):e41.

2. CDC COVID-19 Response Team. Severe outcomes among patients with coronavirus disease 2019 (COVID-19)—United States, February 12-March 16, 2020. MMWR Morb Mortal Weekly. 2020;69(12):343-46.
3. Arvanitakis Z, Shah RC, Bennett DA. Diagnosis and management of dementia: review. JAMA. 2019;322(16):1589-99.

4. Diagnostic and Statistical Manual of Mental Disorders, 5th ed. Washington, DC: American Psychiatric Association; 2013.

5. Todd S, Barr S, Roberts M, Passmore AP. Survival in dementia and predictors of mortality: a review. Int J Geriatr Psychiatry. 2013;28(11):1109-24.

6. Canadian Institute for Health Information. Dementia in Canada. Ottawa, ON: Canadian Institute for Health Information; 2018.

7. Wang H, Li T, Barbarino P, et al. Dementia care during COVID-19 [Correspondence]. Lancet. 2020;395(10231):1190-91.

8. White DB, Lo B. A framework for rationing ventilators and critical care beds during the COVID-19 pandemic [Viewpoint]. JAMA. 2020;323(18):1773-74. Published online March 27, 2020.

9. Emanuel EJ, Persad G, Upshur R, et al. Fair allocation of scarce medical resources in the time of Covid-19. $N$ Engl J Med. 2020;382:2049-55. Published online March 23, 2020.

10. Biddison ELD, Faden R, Gwon HS, et al. Too many patients ... a framework to guide statewide allocation of scarce mechanical ventilation during disasters. Chest. 2019;155(4):848-54.

11. Conde-Sala JL, Garre-Olmo J, Turro-Garriga O, et al. Factors related to perceived quality of life in patients with Alzheimer's disease: the patient's perception compared with that of caregivers. Int J Geriatr Psychiatry. 2009;24(6):585-94.

12. Rockwood K, Song X, MacKnight C, et al. A global clinical measure of fitness and frailty in elderly people. CMAJ. 2005;173(5):489-95.

13. Rockwood K. Clinical Frailty Scale. Halifax, NS: Dalhousie Medical Research. Available from: https://www.dal.ca/sites/gmr/ our-tools/clinical-frailty-scale.html (accessed April 3 2020).

14. Brown MA, Sampson EL, Jones L, et al. Prognostic indicators of 6-month mortality in elderly people with advanced dementia: a systematic review. Palliat Med. 2013;27(5):389-400.

15. Olde Rikkert MG, Tona KD, Janssen L, et al. Validity, reliability, and feasibility of clinical staging scales in dementia: a systematic review. Am J Alzheimers Dis. 2011;26(5):357-65.

16. Fisk JD, Merry HR, Rockwood K. Variations in case definition affect prevalence but not outcomes of mild cognitive impairment. Neurology. 2003;61(9):1179-84.

17. Arya A, Buchman S, Gagnon B, et al. Pandemic palliative care: beyond ventilators and saving lives. CMAJ. 2020; 192(14):E400-E404.

Correspondence to: Eric E. Smith, MD, MPH, Department of Neurology, University of Calgary, Health Sciences Centre, Room 2941, 3330 Hospital Drive NW, Calgary, AB T2N 1N4 E-mail: Eesmith@ucalgary.ca 